\title{
ASSESSMENT OF PREVALENCE OF DEPRESSION IN CARDIAC PATIENTS AND ITS ASSOCIATION WITH THE USE OF BETA-BLOCKERS AND STATINS
}

\author{
KRUTIKA DESAI ${ }^{1}$, NEHA DIWAN ${ }^{1}$, PERIN DEVI MUDHIGANTI ${ }^{1}$, ANAND V JOSHI ${ }^{2}$, NARENDER BOGGULA ${ }^{1}$, \\ VASUDHA BAKSHI ${ }^{*}$
}

${ }^{1}$ Department of Pharmacy Practice, School of Pharmacy, Anurag Group of Institutions, Venkatapur, Ghatkesar, Telangana, India. ${ }^{2}$ Intensivist, Sunshine Hospital, Gachibowli, Hyderabad, Telangana, India. Email: vasudhapharmacy@cvsr.ac.in

Received: 13 July 2018, Revised and Accepted: 13 August 2018

ABSTRACT

Objective: The objective of the study is to assess the prevalence of depression among patients with cardiovascular disease and its association with the use of $\beta$-blockers and statins.

Methods: This is a prospective observational study conducted at a corporate hospital, Hyderabad, Telangana, India, for a period of 6 months. 250 cardiac patients above 16 years are included in the study. The required data are collected from the patients through direct interview using standard questionnaires and also from patients' respective case sheets. The acquired data are evaluated based on the standard questionnaires Patient Health Questionnaire-9 (PHQ-9) and Beck Depression Inventory-II (BDI-II) scales; used to diagnose the severity of depression in cardiac patients.

Results: Prevalence of minor to major depressive symptoms according to BDI-II was found to be $17.2 \%$. Prevalence of minor to major depressive symptoms according to PHQ-9 was found to be $19.2 \%$. Among male patients, $13 \%$ showed depressive symptoms, whereas among female patients $25 \%$ showed depressive symptoms. Among the patients coadministering beta-blockers and statins, 15\% were depressed according to BDI-II, and 16\% were depressed according to PHQ-9 at visit. After 1 month (first follow-up), the percentage increased by 8\% (for BDI-II)-12\% (for PHQ-9) and remains almost the same at the second follow-up. As per BDI-II and PHQ-9 scores, the percentage of patients with minor to major depression among the patients using only beta-blockers decreased significantly from the time of visit to the second follow-up. The percentage of patients with minor to major depression among the patients using only statins increased significantly from the time of visit to second follow-up.

Conclusion: Prevalence of minor to major depression according to BDI-II was found to be $17 \%$, whereas according to PHQ-9, it was found to be $20 \%$ in patients with cardiovascular disease. Cardiovascular diseases have been more prevalent in men than in women, whereas depressive symptoms have been more prevalent in women than in men. Patients using only $\beta$-blockers showed a decrease in symptoms of depression. Whereas statins have shown to increase the chances of depression slightly which is often negligible, atorvastatin was associated with a higher level of depression when compared to rosuvastatin. Controversies still exist that statins decrease risk of depression.

Keywords: $\beta$-Blockers, Depression, Statins, Cardiovascular diseases, Psychological aspects, Screening.

(C) 2018 The Authors. Published by Innovare Academic Sciences Pvt Ltd. This is an open access article under the CC BY license (http://creativecommons. org/licenses/by/4. 0/) DOI: http://dx.doi.org/10.22159/ajpcr.2018.v11i12.28444

\section{INTRODUCTION}

Depression and heart diseases go hand in hand and are among the most disabling diseases we face [1]. Cardiovascular disease (CVD) patients with depression have a worse outcome than those patients without it [2]. The drugs commonly used by CVD patients, i.e. lipophilic beta-blockers and statins are said to have a relationship with respect to increased risk of depressive symptoms. Especially, metoprolol (lipophilic beta blocker) and atorvastatin (statin) appear to increase the risk of depression according to a few recent studies [3]. Few studies were investigated that the effects of statins specifically on mood have reported mixed findings, with some suggesting an increased risk of depression, while others have found either no association between statin use and mood disturbances or that statins, in fact, may protect against the risk of depression $[3,4]$. Betablockers or statins are chief support of CVD. With depression associated independently with CVD, metoprolol, and statin; it is expected to have a larger impact on patients with all three risk factors. We decided to study this prevalence, in a corporate hospital in Hyderabad as data regarding the same is lacking $[4,5]$.

\section{Link between beta-blockers and depression}

First reported in the 1960s, beta-blocker induced depression was thought to result from the drugs' antagonistic effect on norepinephrine at $\beta 1$ post-synaptic brain receptors [6]. A meta-analysis examined 15 randomized, controlled studies involving 35,000 subjects taking beta- blockers for the treatment of myocardial infarction, heart failure, or hypertension, and demonstrated no statistical difference between beta-blockers and placebo with respect to depression, although betablockers were associated with increased incidence of fatigue and sexual dysfunction. The absolute incidence of depressive symptoms was 6/1000 subjects. The authors concluded that it is important to distinguish fatigue from depression that a temporal association between beta-blocker use and depression does not seem to exist (up to 12 months) $[7,8]$.

\section{Possible link between statins and depression}

It is suspected that hundreds of cases of statin-associated hostility, aggression, irritability, paranoia, homicidal ideation, depression, and suicide are somehow linked to dolichols and altered cell messaging. The main function of dolichol (dolichyl phosphate) is its participation in glycoprotein synthesis, and this lipid probably has no other major role in cellular processes [3]. The decrease in the levels of dolichols or its inhibition in the downstream of the mevalonate pathway may possibly cause depression [9-11].

\section{Objectives}

The objective of the study is to assess the prevalence of depression in cardiac patients. The secondary objective of the study is as follows:

- To assess the severity of CVD and depression among men and women.

- To assess the depression in patients using beta-blockers and statins concomitantly. 
- To assess the depression in patients using only beta-blockers.

- To assess the depression in patients using only statins.

\section{METHODS}

\section{Study protocol}

It is a prospective study conducted for a period of 6 months and about 250 patients, met the study criteria were included in the study. The required data are collected from the patients through direct interview using standard questionnaires and also from patients' respective case sheets. The acquired data are evaluated based on the respective questionnaire scales and are used to measure the severity of depression in cardiac patients. ${ }^{[12-14]}$

Study site

The study was conducted at a corporate hospital in the city of Hyderabad, Telangana, India.

\section{Study design}

It is a prospective observational study, wherein standard questionnaire tools, i.e., Patient Health Questionnaire-9 (PHQ-9) and BDI-II are used to diagnose the severity of depression in cardiac patients. ${ }^{[15,16]}$

\section{Study period}

The study period was 6 months (5 months follow-up period).

\section{Study population}

A total of 250 patients are included in the study, of which 150 patients belonged to the in-patient department and 100 patients belonged to the out-patient department.

\section{Study criteria}

Inclusion criteria

- All the patients above the age of 16 years are included in the study.

- All male and female patients, including pregnant women with cardiac diseases, who are able to answer the given questionnaires are included.

\section{Exclusion criteria}

The following criteria were excluded from the study:

- Patients below the age of 16 years.

- Patients without any cardiac complications.

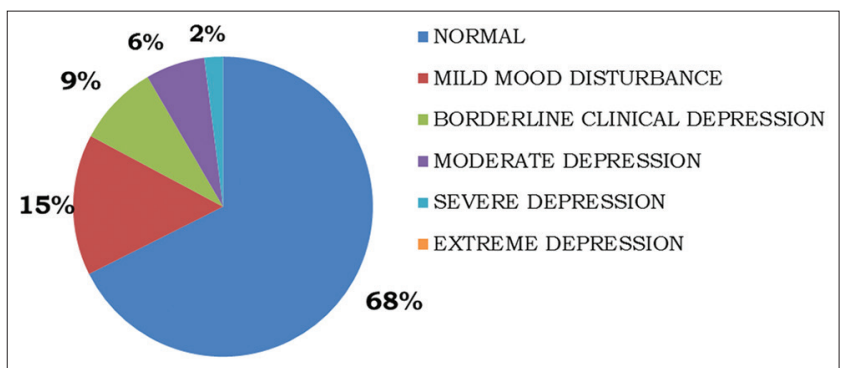

Fig. 1: Distribution based on levels of depression according to the Beck Depression Inventory-II scores

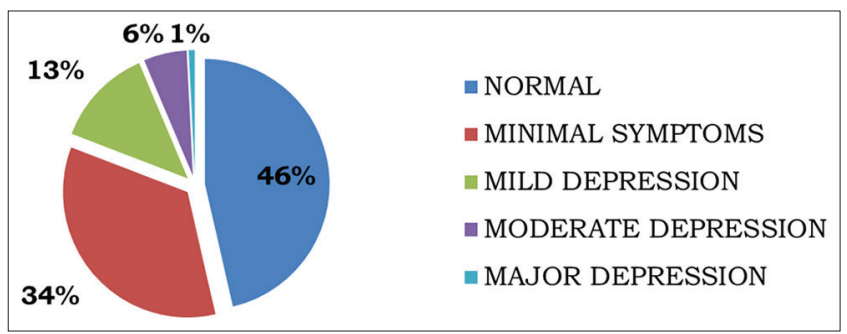

Fig. 2: Distribution based on levels of depression according to Patient Health Questionnaire-9 scores
- Disabled and mentally challenged individuals with or without cardiac complications (who cannot answer the questionnaire).

Two standard questionnaires are taken into consideration to assess depression in cardiac patients which are listed as below.

PHQ-9

The PHQ-9 is a multipurpose instrument for screening, diagnosing, monitoring, and measuring the severity of depression. This is a nineitem questionnaire which helps both to diagnose depression and assess severity Table 1

\section{Beck Depression Inventory-second edition (BDI-II)}

Is a 21-question multiple choice self-report inventory, one of the most widely used psychometric tests for measuring the severity of depression.

\section{Interpreting the BDI-II}

Add up the score for each of the 21 questions by counting the number to the right of each question you marked Table 2 .

\section{RESULTS AND DISCUSSION}

Prevalence of depression in cardiac patients

- Prevalence of minor to major depressive symptoms according to BDI-II was found to be $17.2 \%$.

- Prevalence of minor to major depressive symptoms according to PHQ-9 was found to be $19.2 \%$

- Among male patients, $13 \%$ showed depressive symptoms, whereas among female patients, $25 \%$ showed depressive symptoms.

\section{Distribution based on BDI-II scores}

A total of 250 cardiac patients were screened for depression using BDI-II and PHQ-9. According to BDI-II scores, of 250 patients, $68 \%$ were normal, $15 \%$ showed mild mood disturbances, $9 \%$ had borderline clinical depression, $6 \%$ were moderately depressed, and $2 \%$ suffered from severe depression. Prevalence of borderline to severe depression in cardiac patients according to the BDI-II scores was found to be $17 \%$ as shown in Fig. 1 .

\section{Distribution based PHQ-9 scores}

According to PHQ-9 scores, of 250, 46 were normal, 34\% showed minimal symptoms, $13 \%$ had mild depression, $6 \%$ were moderately

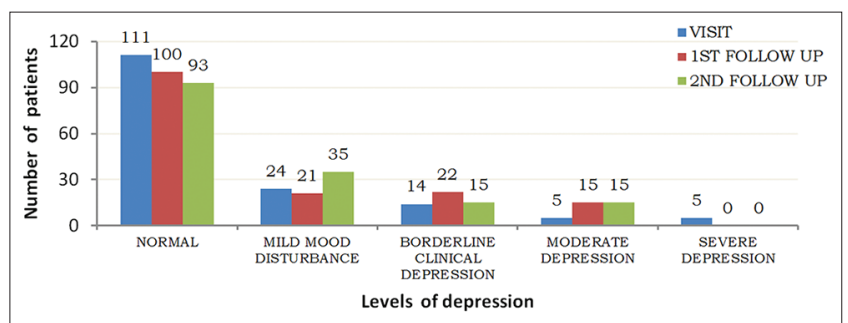

Fig. 3: Distribution of patients using beta-blockers plus statins based on Beck Depression Inventory-II scores

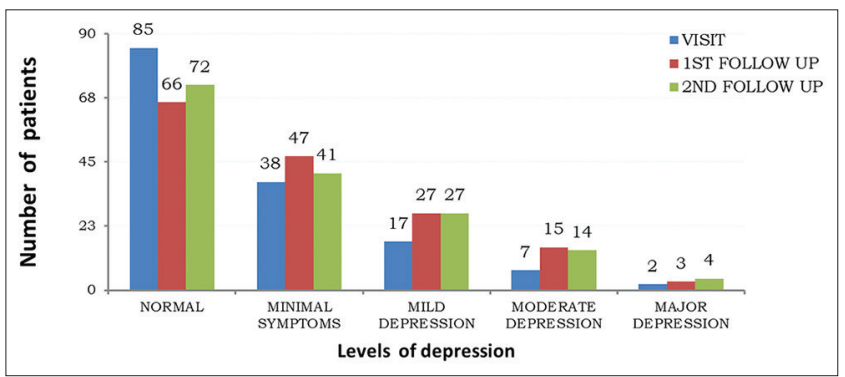

Fig. 4: Distribution of patients using beta-blockers plus statins based on the Patient Health Questionnaire-9 scores 
depressed, and 1\% was found to have major depression. Prevalence of mild to major depression in cardiac patients according to PHQ-9 scores was found to be $20 \%$ as shown in Fig. 2 .

Distribution based on beta-blockers and statins use

Of 250 patients 159 used beta-blockers plus statins concomitantly. From the time of visit to the second follow-up, the number of patients suffering from depression increased. It is clear from the graph that the number of patients who were normal at the visit decreased from 111 at visit to 93 on second follow-up (after 2 months). Number of people with mood disturbances increased, this may be a sign that the condition may further lead to an increase in the severity of depressive symptoms. For patients with moderate depression, the number has increased from 5 patients at visit to 15 patients on the second follow-up as shown in Fig. 3

According to PHQ-9 scores, number patients with minimal to moderate depression are highest during the first follow-up. Number of patients with major depression has doubled from the time of the visit to the second follow-up as shown in Fig. 4.

Prevalence of minor to major depression among the patients using beta-blockers plus statins

Among the patients coadministering beta-blockers and statins, 15\% were depressed according to BDI-II, and 16\% were depressed according to PHQ-9 at visit. After 1 month (first follow-up), the percentage increased by $8 \%$ (for BDI-II)-12\% (for PHQ-9) and remains almost the same at the second follow-up (Fig. 5).

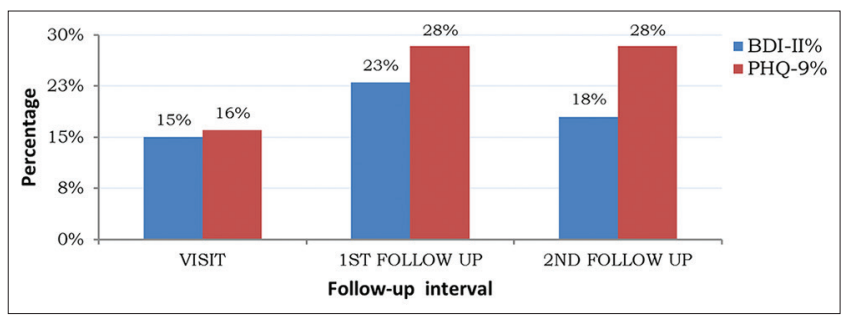

Fig. 5: Percentage of patients with minor to major depression among the patients using beta-blockers plus statins

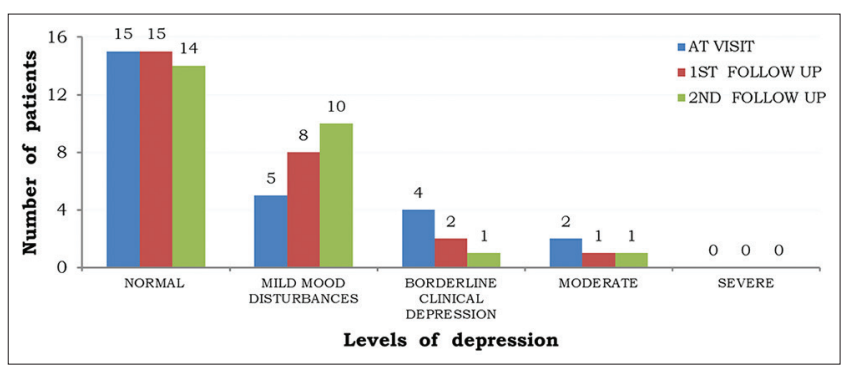

Fig. 6: Distribution of patients using only beta-blockers based on the Beck Depression Inventory-II scores

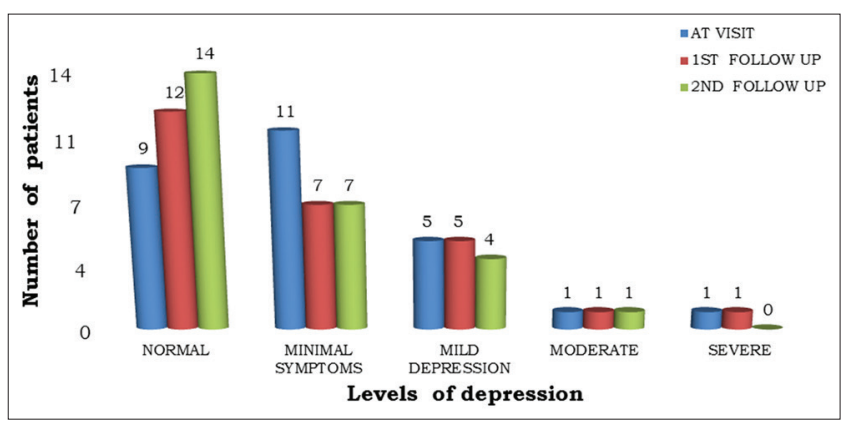

Fig. 7: Distribution of patients using only Beta-blockers based on the Patient Health Questionnaire-9 scores
Distribution of patients based on beta-blockers use

A total of 26 patients used only beta-blockers. Among these patients 15 patients were normal, patients with mood disturbances increased from 5 patients at visit to 10 patients during the second follow-up. There was a decrease in a number of patients with mild to moderate depression from the time of visit to second follow-up as shown in Fig. 6.

According to PHQ-9 scores, in patients using only beta-blockers, there was a decrease in the number of patients with minor to major depression from the time of visit to second follow-up as shown in Fig. 7.

Comparison of BDI-II and PHQ-9 scores in the patients using beta-blockers

As per BDI-II and PHQ-9 scores, the percentage of patients with minor to major depression among the patients using only beta-blockers decreased significantly from the time of visit to the second follow-up as shown in Fig. 8

Percentage of patients with minor to major depression based on the beta-blocker used

Prevalence of depression in patients using metoprolol has increased from the time of visit to first follow-up and decreased again in the second follow-up, while in carvedilol users it was a quiet contrast scenario with decreased prevalence of depressive symptoms at first

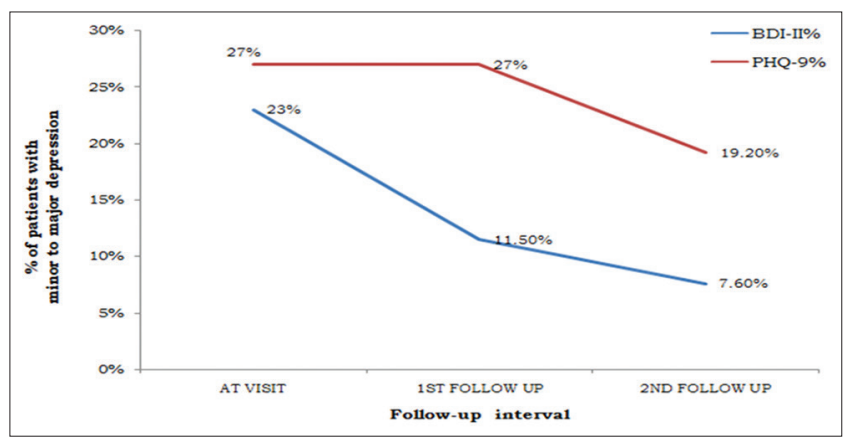

Fig. 8: Comparison of Beck Depression Inventory-II and Patient Health Questionnaire-9 scores in the patients using beta-blockers

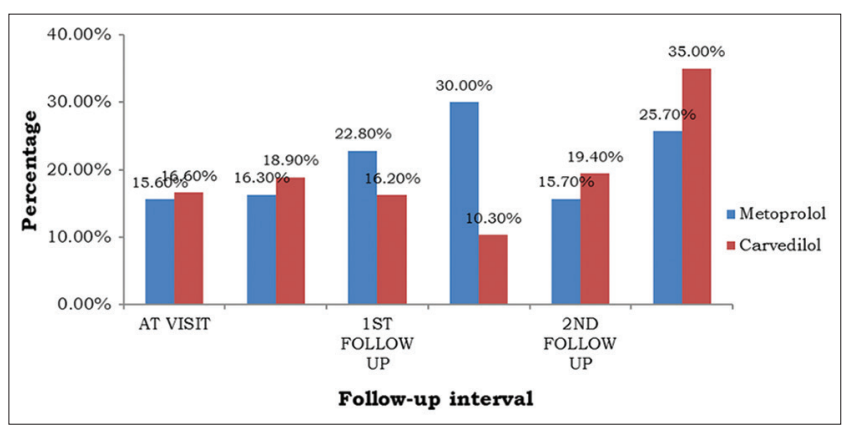

Fig. 9: Percentage of patients with minor to major depression based on the beta-blocker used

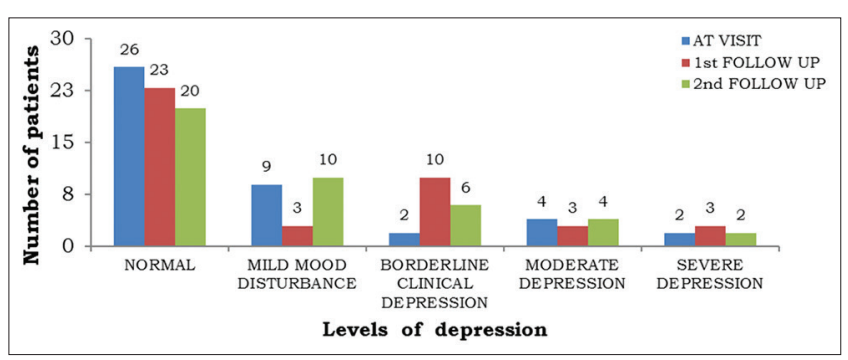

Fig. 10: Distribution of patients using only statins based on the Beck Depression Inventory-II scores 
follow-up and a steep rise in prevalence was observed at the second follow-up as shown in Fig. 9.

\section{Distribution based on statins use}

A total of 43 patients used only statins. According to the BDI-II scores, in patients using only statins, there was no significant difference between the number of patients with moderate to severe depression from the time of visit to second follow-up. However, there was a slight increase in number patients with borderline clinical depression or mild depression from visit to second follow-up as shown in Fig. 10.

There was an increase in moderate - major depression from the time of visit to the second follow-up, whereas number of patients with minimal symptoms and mild depression decreased from the time of visit to the second follow-up as shown in Fig. 11.

\section{Comparison of BDI-II and PHQ-9 score in the patients using only} statins

The percentage of patients with minor to major depression among the patients using only statins increased significantly from the time of visit to second follow-up as shown in Fig. 12.

\section{Distribution based on the type of statins}

From the time of the visit to the second follow-up, the percentage of patients with depression has increased from $16.6 \%$ to $22.7 \%$ according to BDI-II score in case of atorvastatin users and 12.1-15.60\% according to PHQ-9 score in case of rosuvastatin users. It is clear that the prevalence of depression in atorvastatin users is higher than that of rosuvastatin users as shown in Fig. 13.

Screening for depression was done using the two standard questionnaires. Throughout the study period, on an average $17.2 \%$ of the whole study population were found to exhibit symptoms of depression based on BDI-II score. In accordance with the PHQ-9 scores, $19.2 \%$ of total study population exhibited minor to major depression. Almost about $3 / 4^{\text {th }}$ of the population (i.e., $73 \%$ ) hold to be males, and the rest $1 / 4^{\text {th }}($ i.e., $27 \%$ ) were females. Therefore, it's a clear indication that males are more affected by cardiovascular diseases as compared to females. The reason for this might be several lifestyle factors that differ in men and women. Smoking has been the paramount risk factor for CVD and men are more prone to the habit of smoking than women, in a country like India. This might be one of the reasons why the study showed that a high percentage of men with coronary artery disease. Hence, it is assumed that exposure to endogenous estrogens during the fertile period of life delays the manifestation of atherosclerotic disease in women.

Depression is approximately twice as prevalent among women as it is among men. The results in the present study show that on an average 23 of 68 females (33\%) and 42 of 182 males (23\%) have observed to have minor-major depression (BDI-II score >16; PHQ-9 score $>9$ ). Thus, it conveys that females are 1.5 times more affected with depression than males. Many have attributed this disparity to the various stresses women face as a result of their gender and the demands women confront as they occupy multiple and often conflicting roles within the family, in the community, and at work.

Our study predicates that concomitant use of beta-blockers and statins may increase the chances of having depression. Although no evidence of such interaction has been mentioned in previous studies, the statement is completely contingent on our observations in the study population. Further, studies in this aspect may provide us with better evidence.

Patients using beta-blockers experienced a reduction in depression during the course of the study, but most of them had mild mood disturbances after a 3 month follow-up. In connection to the above observations, statins might not show a greater degree of variability in psychological aspects of a patient. Yet, slight increase in depressive symptoms seems to occur with statin use which may often be overlooked. A pile of studies were done by researchers in relation to this facet but

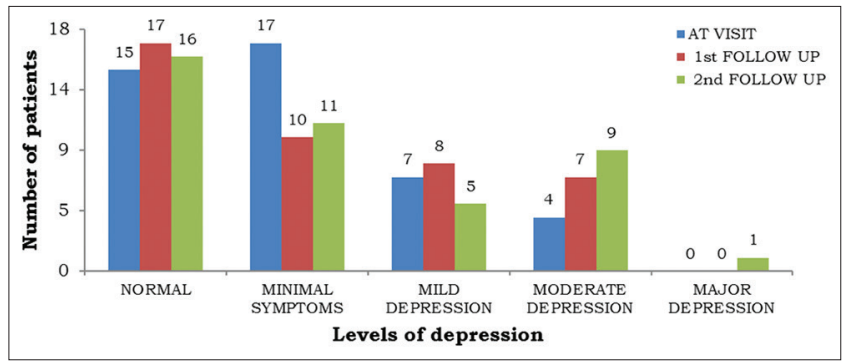

Fig. 11: Distribution of patients using only statins based on the Patient Health Questionnaire-9 scores

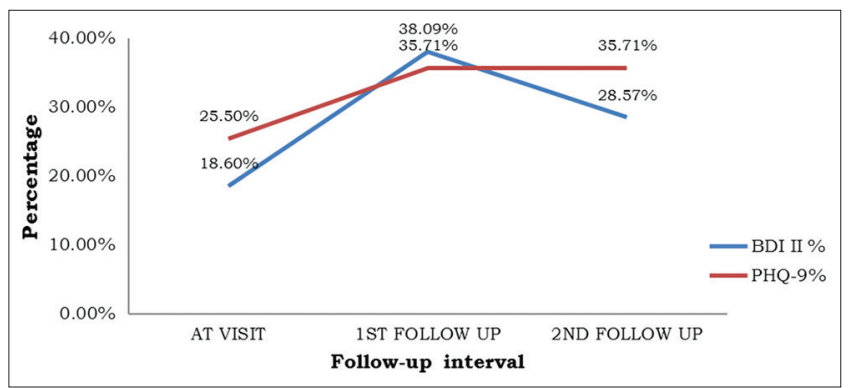

Fig. 12: Comparison of Beck Depression Inventory-II and Patient Health Questionnaire-9 score in the patients using only statins

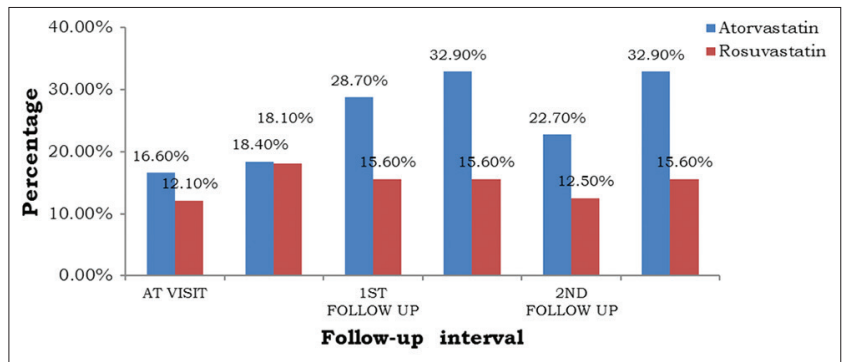

Fig. 13: Percentage of patients with minor to major depression based on the statins used

failed to establish a clear association between statins and depression. Researchers have put forth number of hypotheses; one such hypothesis affirms that depression in patients using beta-blockers may be due to the cholesterol-lowering effect of statins in brain. Individuals who take statins to lower their cholesterol sometimes display symptoms of irritability, anxiety, and depression; these symptoms are reported by their family members, caregivers, and coworkers.

\section{CONCLUSION}

- Prevalence of minor to major depression according to BDI-II was found to be $17 \%$.

- Prevalence of minor to major depression according to PHQ-9 was found to be $20 \%$.

- Cardiovascular diseases have been more prevalent in men than in women whereas depressive symptoms have been more prevalent in women than in men.

- Use of beta-blockers and statins concomitantly may lead to a slight increase in depressive symptoms. Therefore, in patients who are on both the medications, there is a need for screening of depressive symptoms and monitor the patients' status from time to time.

- The study reveals that the use of beta-blockers is not associated with an increase in depression. In fact, it has shown to decrease the symptoms of depression in cardiac patients who were on betablocker therapy. 
Table 1: PHQ-9 score scale

\begin{tabular}{|c|c|c|}
\hline PHQ-9 score & Provisional diagnosis & $\begin{array}{l}\text { Treatment } \\
\text { recommendation patient } \\
\text { preferences should be } \\
\text { considered }\end{array}$ \\
\hline $5-9$ & Minimal symptoms & $\begin{array}{l}\text { Support, educate to call if } \\
\text { worse, return in } 1 \text { month }\end{array}$ \\
\hline 10-14 & $\begin{array}{l}\text { Minor depression, } \\
\text { dysthymia, major } \\
\text { depression, and mild }\end{array}$ & $\begin{array}{l}\text { Support, watchful } \\
\text { waiting, antidepressant } \\
\text { or psychotherapy, } \\
\text { and antidepressant or } \\
\text { psychotherapy }\end{array}$ \\
\hline $15-19$ & $\begin{array}{l}\text { Major depression, and } \\
\text { moderately severe }\end{array}$ & $\begin{array}{l}\text { Antidepressant or } \\
\text { psychotherapy }\end{array}$ \\
\hline$>20$ & $\begin{array}{l}\text { Major depression, } \\
\text { severe }\end{array}$ & $\begin{array}{l}\text { Antidepressant or } \\
\text { psychotherapy (especially } \\
\text { if not improved on } \\
\text { monotherapy) }\end{array}$ \\
\hline
\end{tabular}

PHQ-9: Patient Health Questionnaire-9

Table 2: Level of depression

\begin{tabular}{ll}
\hline Total score & Level of depression \\
\hline $0-10$ & Normal \\
$11-16$ & Mild mood disturbance \\
$17-20$ & Borderline clinical depression \\
$21-30$ & Moderate depression \\
$31-40$ & Severe depression \\
$>40$ & Extreme depression \\
\hline
\end{tabular}

- Use of statins was observed to increase the chances of depression slightly which is often neglected as the symptoms may not be severe and may be often under-diagnosed.

- Atorvastatin was associated with a higher level of depression when compared to Rosuvastatin.

- Screening of the depression in cardiovascular patients should be made obligatory as its prevalence is significant.

\section{ACKNOWLEDGMENT}

The authors wish to thank the management of School of Pharmacy, Anurag Group of Institutions, Venkatapur, Ghatkesar, Telangana, India, for providing necessary equipment for research, constant encouragement, facilities, and support. We personally thank Dr. Anand V Joshi, Intensivist, Sunshine Hospital, Gachibowli, Hyderabad, without whom the research work would not have been possible in the way it has been completed. We feel privileged for his constant guidance, motivation, and encouragement, for facilitating, sequencing our approach in the research work, and for all the requirements. We also thank all the hospital staff for all their cooperation extended in this research.

\section{AUTHOR'S CONTRIBUTION}

All authors contributed to the design and implementation of the research, to the analysis of the results and the writing of the final manuscript. All authors provided critical feedback and helped shape the research, analysis, and manuscript.

\section{CONFLICTS OF INTEREST}

The authors declare that there are no conflicts of interest.

\section{REFERENCES}

1. Vaccarino V, Bremner JD. Braunwald's Heart Disease. A Textbook of Cardiovascular Medicine, Psychiatric and Behavioral Aspects of Cardiovascular Disease. $9^{\text {th }}$ ed. Philadelphia: Saunders; 2012.

2. Xiong GL, Gagliardi JP, Jiang W. Beta-blockers and depression. Am J Psychiatry 2010;167:219.

3. Chojnacki T, Dallner G. The biological role of dolichol. Biochem J 1988;251:1-9.

4. Redlich C, Berk M, Williams LJ, Sundquist J, Sundquist K, Li X, et al. Statin use and risk of depression: A Swedish national cohort study. BMC Psychiatry 2014;14:348.

5. Katsarou A, Triposkiadis F, Skoularigis J, Griva E, Neroutsos G, Karayannis G, et al. Evaluating the role of perceived stress on the likelihood of having a non-fatal acute coronary syndrome: A casecontrol study. Open Cardiovasc Med J 2014;8:68-75.

6. McGuire AW, Ahearn E, Doering LV. Psychological distress and cardiovascular disease. J Clin Outcomes Manag 2015;22:421-32.

7. Brigitta B. Pathophysiology of depression and mechanisms of treatment. Dialogues Clin Neurosci 2002;4:7-20.

8. Bonfim MR, Oliveira AS, do Amaral SL, Monteiro HL. Treatment of dyslipidemia with statins and physical exercises: Recent findings of skeletal muscle responses. Arq Bras Cardiol 2015;104:324-31.

9. Colin A, Reggers J, Castronovo V, Ansseau M. Lipids, depression and suicide. Encephale 2003;29:49-58

10. Bivanco-Lima D, Souza Santos Id, Vannucchi AM, Almeida Ribeiro MC. Cardiovascular risk in individuals with depression. Rev Assoc Med Bras (1992) 2013;59:298-304.

11. Koul RK, Parveen S, Lahdol P, Rasheed S, Shah NA. Prevalence and risk factors of gastroesophageal reflux disease (GERD) in adult Kashmiri population. Int J Pharm Pharm Sci 2018;10:62-6.

12. Paz-Filho G, Licinio J, Wong ML. Pathophysiological basis of cardiovascular disease and depression: A chicken-and-egg dilemma. Rev Bras Psiquiatr 2010;32:181-91.

13. Sripada R, Kumar SV, Devanna N, Reddy KR. A study on the prevalence and severity of possible drug-drug interactions in pediatrics department at an Indian tertiary care teaching hospital. Int J Pharm Pharm Sci 2018;10:52-4

14. Gottlieb SS, Khatta M, Friedmann E, Einbinder L, Katzen S, Baker B, et al. The influence of age, gender, and race on the prevalence of depression in heart failure patients. J Am Coll Cardiol 2004;43:1542-9.

15. Hodgin JB, Krege JH, Reddick RL, Korach KS, Smithies O, Maeda N, et al. Estrogen receptor alpha is a major mediator of 17beta-estradiol's atheroprotective effects on lesion size in apoe-/- mice. J Clin Invest 2001;107:333-40.

16. Prakruthi GM, Bharathi DR, Yogananda R. A case control study on determinants of childhood asthma in school children of Chitradurga city. Int J Curr Pharm Res 2018;10:39-43 